\title{
Evaluation of the Efficacy of Subconjunctival Anti Vascular Endothelial Growth Factor Antibody Injection in the Treatment of Retinopathy of Prematurity in an Experimental Rat Model
}

\author{
Tuncay Topal ${ }^{1 *}$, Yakup Aksoy ${ }^{1}$ and Ali Ayata ${ }^{2}$ \\ ${ }^{1}$ Specialist, Department of Ophthalmology, Haydarpasa Sultan Abdulhamid \\ Training and Research Hospital, Üsküdar, Istanbul, Turkey \\ ${ }^{2}$ Associated Professor, Department of Ophthalmology, Haydarpasa Sultan \\ Abdulhamid Training and Research Hospital, Üsküdar, Istanbul, Turkey \\ *Corresponding Author: Tuncay Topal, Specialist, Department of Ophthalmology, \\ Haydarpasa Sultan Abdulhamid Training and Research Hospital, Üsküdar, Istanbul, \\ Turkey.
}

Received: September 26, 2020

Published: October 07, 2020

(C) All rights are reserved by Tuncay Topal., et al.

\section{Abstract}

Purpose: To observe the effect of subconjunctival (SC) approach for the treatment of retinopathy of prematurity (ROP) in an experimental rat model.

Methods: We used 4 groups (G) comprising 18 new-born rats in each. G1: healthy control, G2: untreated oxygen induced retinopathy (OIR) control, G3: SC rat anti vascular endothelial growth factor antibody (antiVEGFab) injected and G4: Intravitreal (IVT) antiVEGFab injected. G2, G3 and G4 were exposed to oxygen fluctuations alternating between 50\% and 10\% every 24 hours until postnatal day (p) 14 at which time the groups were placed into room air for 4 days. At p18; OIR was observed through fluorescein angiography (FA). G3 and G4 were treated immediately $1 \mu \mathrm{g} / 10 \mu \mathrm{l}$ and $50 \mathrm{ng} / 2 \mu \mathrm{l}$ respectively. FAs repeated at p19, p25 and p30. To evaluate 0IR; modified retinopathy scoring system (MRSS) was calculated according to the FA images, including retinal vascular development, vascular tuft formation, extraretinal neovascularization, vascular tortuosity and retinal haemorrhage. Blood analysis was performed on the same three days for the measurement of serum VEGF levels. Histopathological evaluation was performed with haematoxylin \& eosin staining for the vascular lumen quantification on the retinal specimens of the enucleated eyes on the same days.

Results: There was no significant difference between the G3 and G4 at the end of the study according to the MRSS values(p: 0,101), systemic VEGF levels (p: 0,796) and vascular lumen counts of histopathological specimens (p: 0,359).

Conclusion: The therapeutic effect of SC and IVT on OIR were similar. SC anti VEGF treatment may be a safer and effective alternative to IVT injection for the treatment of ROP.

Keywords: Subconjunctival; Retinopathy of Prematurity; Vascular Endothelial Growth Factor; Oxygen Induced Retinopathy

\section{Introduction}

VEGF plays an essential role in the vasoproliferative process in ROP. It is secreted from Müller cells and astrocytes in response to hypoxia caused by hypoperfusion in the retinal layers. Inhibition of VEGF bioactivation is a key target in the treatment of ROP [1]. The first attempt in ROP treatment was the ablation of VEGF-producing retinal avascular areas. For this, cryotherapy was first used in 1980s. Laser photocoagulation has taken the place of cryotherapy since the 1990s and has become more widespread over time, is now the gold standard treatment [2]. However, the long-term complications of this treatment, such as visual field defects, high myopia, and difficulties in implementation have prompted ophthalmologists to seek new treatments [3]. In an ideal treatment protocol, vital retinal fields should be protected, the pathological 
Evaluation of the Efficacy of Subconjunctival Anti Vascular Endothelial Growth Factor İnjection in the Treatment of Retinopathy of Prematurity in an Experimental Rat Model

vasoproliferative process should be prevented, and physiological retinal vascular maturation must be completed. Anti VEGF Abs have been the most studied drugs for this purpose [4-8]. Although IVT anti-VEGF Ab treatment is effective in the treatment of ROP, it has many complications, such as increased intraocular pressure, lens damage, endophthalmitis, intravitreal haemorrhage, retinal tears and detachment. Another important risk is the possibility that the IVT-injected drug may cause a systemic blockade of VEGF [9].

\section{Aim of the Study}

In this study, we aimed to compare SC and IVT anti-VEGF Ab injections in terms of treatment success and systemic VEGF blockade in an experimental rat model of ROP.

\section{Methods}

This study was approved by Gulhane Military Medical Academy Haydarpasa Training and Research Hospital Animal Experiments Local Ethics Committee with the project number 2016/3. Seventy-two new-born Sprague Dawley rat pups were used. Our study complies with the ARRIVE guidelines and the pups were all handled according to the ARVO statement for the Use of Animals in Ophthalmic and Vision Research. Eighteen of pups were left in normal room air conditions to form a healthy control group. Fifty-for of the pups were placed into a transparent plastic, incubator-like box with their mothers to cause OIR.

Animal model of ROP (Oxygen-induced retinopathy): All of the rats were born at term. At p0, G2, G3 and G4, with their mothers, were placed into the box with cycled oxygen between $50 \%$ and $10 \%$ every 24 hours. At p14, the pups were returned to room air for 4 days. In the indoor environment, the only other gas used other was nitrogen. We used the best strategy of John Penn to create OIR [10].

Fluorescein angiography (FA): A total of 1\% cyclopentolate, $1 \%$ tropicamide and $2.5 \%$ phenylephrine were administered topically for pupil dilation. Then, $0.05 \mathrm{ml} 1 \%$ sodium fluorescein was injected intraperitoneally (IP). For the evaluation of normal retinal vascular development and OIR formation, all groups were subjected to FA (Spectralis Heidelberg, Germany) at p18 (Figure 1). FAs were performed at p19, p25 and p30 to observe normal retinal vascular development, the natural course of OIR, and the response to treatment.

Modified retinopathy scoring system (MRSS): The severity of OIR was evaluated using MRSS on FA images. MRSS was applied using the following five parameters: retinal vascular development, vascular tuft formation, extraretinal neovascularization, vascular tortuosity and retinal haemorrhage. We determined 3 equal zones with the optic disc in the centre. Vascular development was assessed according to these zones. The assessment was performed according to the existence of retinal haemorrhage, while vascular tuft formation, extraretinal neovascularization, and vascular tortuosity were scored according to the clock quadrant involved (Table 1). Bilateral scoring was performed for six rats on each examination day from each group to determine the changes in the same rat eye

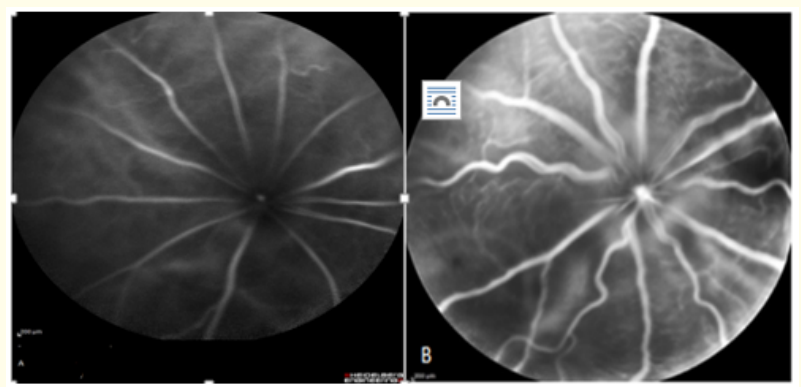

Figure 1: FA image samples of the normal rat retina and the OIRformed rat retina at $\mathrm{p} 18$. (A) In the normal rat retina, 12 vessels came out of the optic disc and progressed radially to the periphery of the retina in a regular manner. (B) In the OIR rat retina, increased arterial tortuosity and increased venous filling were observed which is similar to the appearance of human ROP.

over time. MRSS was applied by the same clinician to all eyes. . Retinopathy scoring methods similar to the ones we use have been performed in some previous studies [11-14].

Rat anti-VEGF ${ }_{164}$ Ab injection: A total of 0.5\% proparacaine and $20 \%$ diluted povidone were administered topically for local anaesthesia and antisepsis. The rat anti-VEGF ${ }_{164} \mathrm{Ab}$ (R and D Systems, Minneapolis, MN) was injected bilaterally. The drug doses were $1 \mu \mathrm{g} / 10 \mu \mathrm{l}$ for the SC injection and $50 \mathrm{ng} / 2 \mu \mathrm{l}$ for the IVT injection. A Hamilton injector and 34-gauge needles were used for both injections. The SC injections were administrated from any conjunctival area. The IVT injections were administered just posterior to the limbus to avoid lens damage. Then, $0.3 \%$ netilmicin was applied to the injected eyes.

Blood sampling and sacrificing: At p19, p25 and p30, six rat pups from each group were exposed to general anaesthesia with ether. A maximum volume of blood was drawn intracardiacally with a 1-ml 30-gauge tuberculin syringe. Thus, both blood sampling and 
Evaluation of the Efficacy of Subconjunctival Anti Vascular Endothelial Growth Factor İnjection in the Treatment of Retinopathy of Prematurity in an Experimental Rat Model

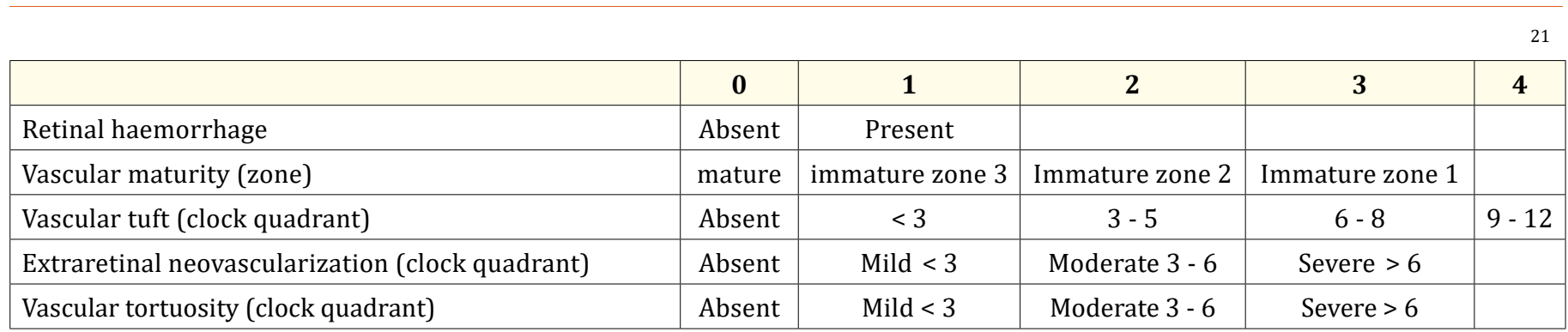

Table 1: Modified retinopathy scoring system.

sacrificing were performed at the same time. The blood samples were centrifuged to separate the serum and stored at $-80^{\circ} \mathrm{C}$ for rat VEGF $_{164}$ measurements with an ELISA (SunRed) kit.

Enucleation and histopathological evaluation: Bilateral enucleation was performed by dissecting the eyes from the optic nerve and surrounding intraorbital tissues after sacrificing. After enucleation, the eyes were left in a $4 \%$ paraformaldehyde (PFA) solution for 2 hours. Ten specimens from each eye were prepared and stained with haematoxylin and eosin. The vascular lumen counts for each specimen were calculated, and the average value for each eye was determined. The vascular lumen counts were conducted by the same researcher on all eyes.

Statistical analysis: All statistical analyses were performed with SPSS (Statistical Package for the Social Sciences version 17.0.0; SPSS Inc., Chicago, IL, USA). Friedman test was conducted to assess whether there were significant changes in the VEGF levels, vascular lumen counts and MRSS values. The Wilcoxon test was performed to test the significance of pairwise differences using Bonferroni correction to adjust for multiple comparisons. Kruskal Wallis test was used to compare VEGF levels, vascular lumen counts and MRSS values among the groups. Mann-Whitney U test was performed to test the significance of pairwise differences using Bonferroni correction. An overall, 5\% type 1 error level was used to infer statistical significance.

\section{Results}

Changes in MRSS values at p18, p19, p25 and p30 in each group (Graph 1)

There was no statistically significant decrease in the MRSS values in G2 over time (p: 0.004). The comparisons were: p18-p19 (p: 0.169), p18-p25 (p: 1.000), p19-p25 (p: 0.085), p19-p30 (p: 0.150), p18-p30 (p: 0.043) and p25-p30 (p: 0.036) (Figure 2).

A statistically significant decrease in the MRSS values was observed in G3 ( $\mathrm{p}<0.001)$. The comparisons were: p18-p19 (p:

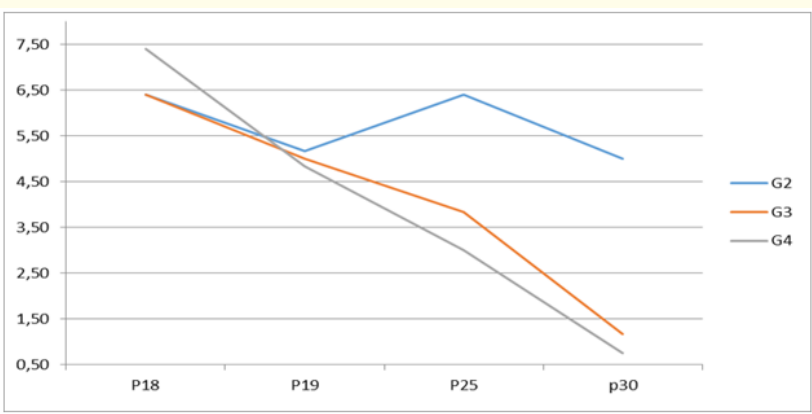

Graph 1: MRSS values in the groups over course of time.

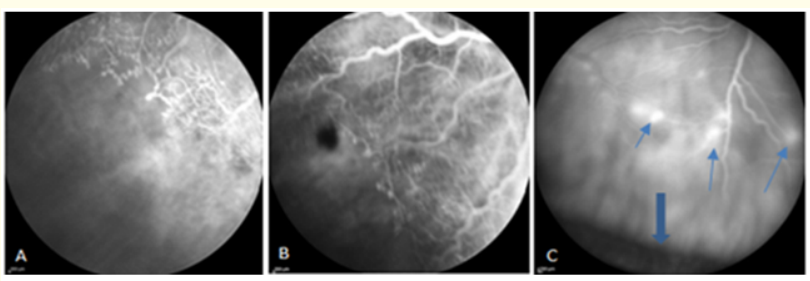

Figure 2: FA images of time-dependent changes in the same retinal area of an untreated rat in G2. (A) At p19, a wide avascular area and vascular tufts were seen at the border of the vascular - avascular region. (B) At p25, a resolution began, a prevascular area was vascularized, a retinal haemorrhage developed at one point, venous filling was increased and tortuosity was present. (C) At p30, the vascularization did not reach the ora serrata, and some areas of NV had formed. The thin arrows in C indicate NVs, and the thick arrow indicates the ora serrata.

0.004), p18-p25 (p: 0.002), p18-p30 (p: 0.002), p19-p30 (p: 0.002) and p25-p30 (p: 0.002). However, the decrease in the p19-p25 (p: 0.030 ) range was not significant (Figure 3).

A statistically significant decrease in the MRSS values also observed in G4 ( $p<0.001)$. The comparisons: p18-p19 (p: 0.002), 


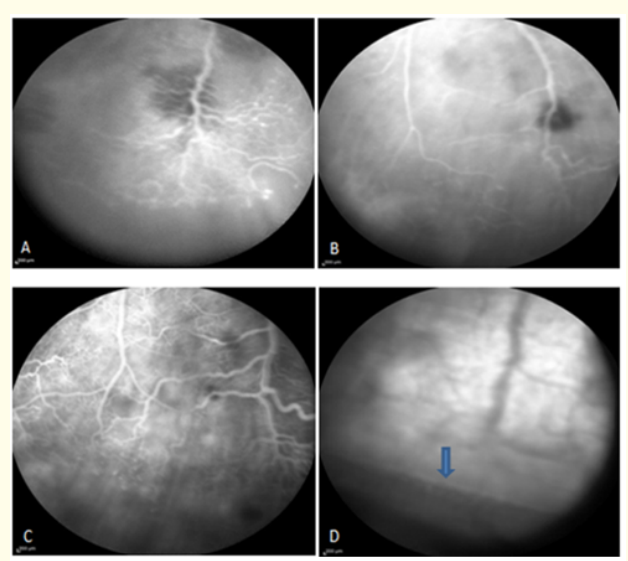

Figure 3: FA images of time-dependent changes in the same retinal area of an SC-treated rat in G3. (A) At p18, a retinal hemorrhage was present in the OIR model with, peripheral avascular space, mild tortuosity in the vascular structures, and vascular tufts at the border of the avascular-vascular space. (B) At p19, one day after the SC injection, the retinal haemorrhage dimensions and tortuosity decreased. (C) At p25, retinal hemorrhage was much smaller, the vascular area was wider, and the NV and vascular tufts regressed. (D) At p30, the vascularization reached the ora serrata (thick arrow).
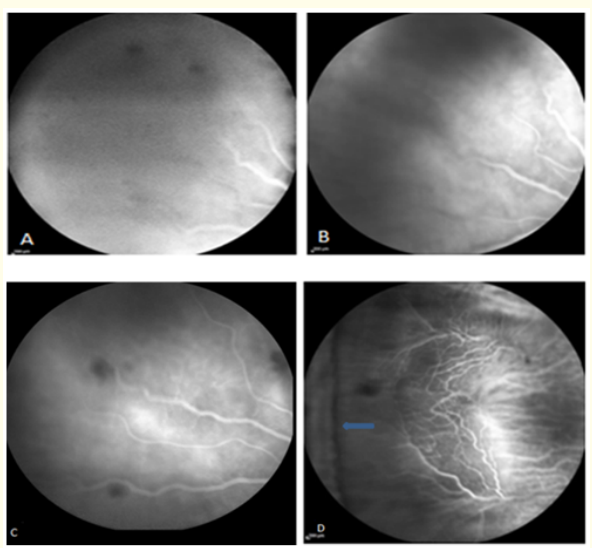

Figure 4: FA images of the time course of the same retinal area of an IVT treated rat in G4. (A) At p18, - a large avascular area. (B) At p19 - less avascular space. (C) At p25 - the avascular area was further reduced, and two small haemorrhagic areas were observed. (D) At p30 - the vascular structures were advanced to the ora serrata (thick arrow). p18-p25 (p: 0.003), p18-p30 (p: 0.002), p19-p30 (p: 0.002) and p25-p30 (p: 0.005). However, the decrease in the p19-p25 (p: 0.012 ) range was not significant (Figure 4).

Comparison of the MRSS values between the groups at p18, p19, p25 and p30 (Graph 1)

At p25, the MRSS values of G2 were significantly higher than those of both G3 and G4 (p < 0.001, p < 0.001, respectively). However, there was no significant difference between G3 and G4 (p: $0.100)$.

The severity of retinopathy in G2 at p30 was significantly higher than that in both G3 ( $p<0.001)$ and G4 (p < 0.001). No significant difference was observed between G3 and G4 (p: 0.101).

Changes in the serum VEGF levels at p19, p25 and p30 in each group (Graph 2)

There was a statistically significant decrease in the G1 serum VEGF levels over time (p: 0.006). All reductions in p19-p25 (p: 0.028), p19-p30 (p: 0.028) and p25-p30 (p: 0.046) were statistically significant.

There was a statistically significant decrease in the G2 serum VEGF levels over time (p: 0.002). All reductions in p19-p25 (p: 0.028), p19-p30 (p: 0.028) and p25-p30 (p: 0.028) were statistically significant.

There was a statistically significant decrease in the G3 serum VEGF levels over time (p: 0.002). The reductions in p19-p25 (p: 0.012) and p19-p30 (p: 0.012) were statistically significant, but a slight increase between the p25-p30 interval was not statistically significant (p: 0.779).

There was a statistically significant decrease in the G4 serum VEGF levels over time (p: 0.009). The reductions in the p19-p25 (p: 0.012) and p19-p30 (p: 0.028) were statistically significant, but a slight increase between p25-p30 interval was not statistically significant (p: 0.345).

Comparison of the serum VEGF levels between the groups at p19, p25 and p30 (Graph 2)

At 1 19; the systemic VEGF levels in G1 were significantly lower than G2 (p: 0.004), G3 (p: 0.002) and G4 (p: 0.004). The VEGF levels between G2-G3 (p: 0.014), G2-G4 (p: 0.749) and G3-G4 (p: 0.197) were not statistically significant.

At p25; the systemic VEGF levels in G1 were statistically signi- 
Evaluation of the Efficacy of Subconjunctival Anti Vascular Endothelial Growth Factor İnjection in the Treatment of Retinopathy of Prematurity in an Experimental Rat Model

ficantly lower than those in G2 (p: 0.004) but significantly higher than those in G3 (P: 0.005) and G4 (p: 0.004). The VEGF levels in G2 were significantly higher than those in G3 (p: 0.002) and G4 (p: 0.004). There was no significant difference between G3 and G4 (p: $0.796)$.

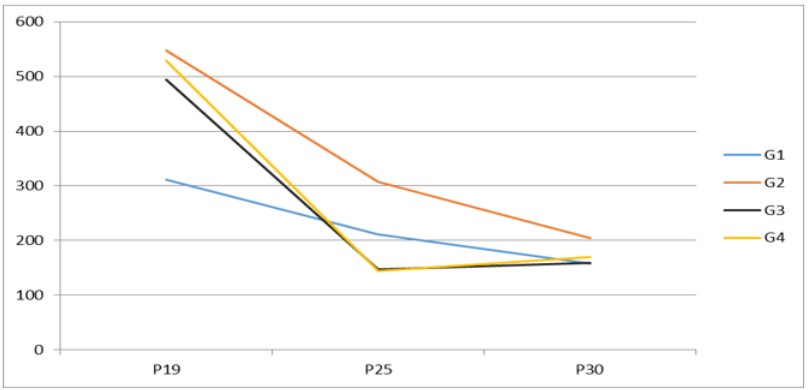

Graph 2: The VEGF levels (pg/ml) in the groups over the course of time.

At p30, there was no significant difference between the 4 groups (p: 0.265).

Changes in the vascular lumen counts in the histopathological specimens at p19, p25 and p30 in each group (Graph 3)

The decrease in G2 was not statistically significant (p: 0.293) (Figure 5).

There was a statistically significant difference in G3 over time. The comparisons; p19-p25 (p: 0.069) and p25-p30 (p: 0.069) were not significant but the decrease in the p19-p30 interval was significant (p: 0.017) (Figure 5).

There was a statistically significant difference in G4 over time. The comparisons; p19-p25 (p: 0.028), p19-p30 (p: 0.028) and p25-p30 (p: 0.046) were significant (Figure 5).

Comparison of the vascular lumen counts between the groups at p19, p25 and p30 (Graph 3)

No significant difference was found between the groups at p19 (p: 0.572) and p25 (p: 0.117) (Figure 5).

At p30, there was a statistically significant difference. The vascular lumen counts in G2 were higher than those in G3, but there was no statistically significant difference (p: 0.032). However, the

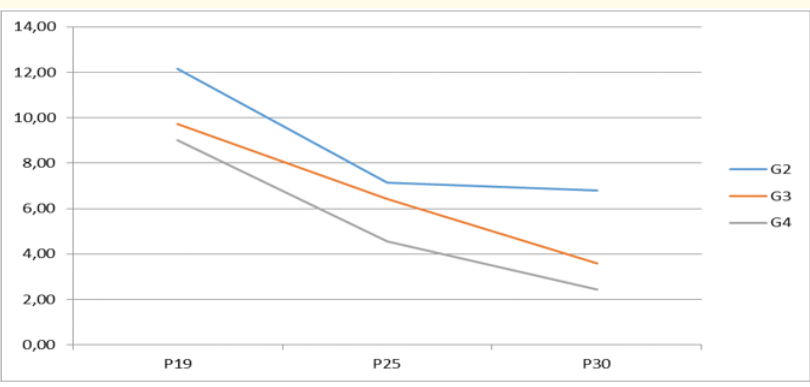

Graph 3: The vascular lumen counts in the groups over the course of time (since this was a pathological assessment, G1 was excluded).

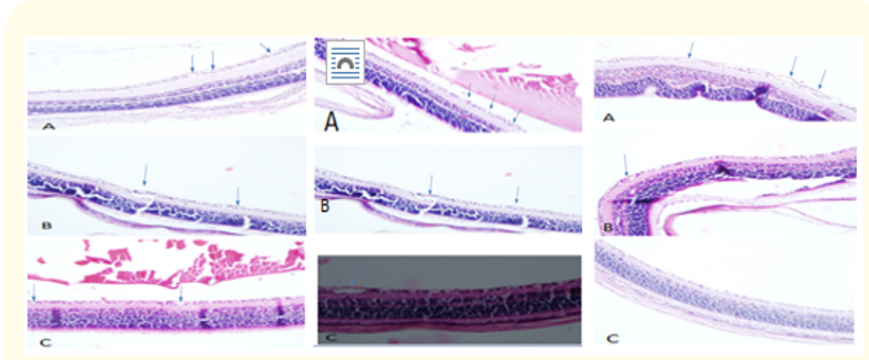

Figure 5: Haematoxylin and eosin stained histopathological sections. The images represent G2, G3, and G4 from left to right and, p19, p25 and p30 from top to bottom. The arrows indicate vascular lumens on the retinal surface.

vascular lumen counts in G2 were significantly higher than those in G4 (p: 0.013). There was no significant difference between G3 and G4 at p30 (p: 0.359) (Figure 5).

\section{Discussion}

The experimental animal model, which is the best recapitulation of the human ROP, is the 50/10 model developed by John Penn [10]. In this model, the changes seen over time are as follows. At $\mathrm{p} 12$, the increase in vascular tortuosity is similar to that in human disease. At p14, retinal VEGF reached the maximal levels and large avascular areas appeared, which accounted for approximately $30 \%$ of the retinas. At p18, a significant increase in VEGF levels in the vitreous occurred, which was consistent with human stage 3 ROP, and led to the growth of the vessels away from the retinal surface and towards the vitreous. At p25, the intravitreal neovascularization (NV) reached the maximum levels, and the OIR began to resolve. 
Evaluation of the Efficacy of Subconjunctival Anti Vascular Endothelial Growth Factor İnjection in the Treatment of Retinopathy of Prematurity in an Experimental Rat Model

At p30, the complete resolution of OIR was observed. These findings have been confirmed by several studies [11-14]. We constructed the rat OIR model, planned the follow-up scheme and the methodology of our study in accordance with these data.

Geisen., et al. [15] showed that a 50-ng IVT injection of rat anti-VEGF ${ }_{164}$ Ab blocked NV and did not disrupt the physiological retinal vascularization, $50 \mathrm{ng}$ of the Ab was defined as a neutralization dose of rat anti-VEGF ${ }_{164} \mathrm{Ab}$. This was also confirmed by Hartnett., et al [12]. Since we performed the IVT injections with $50 \mathrm{ng}$ doses.

The main purpose of the IVT anti-VEGF Ab injection in ROP therapy is to reduce the VEGF levels in the vitreous and to direct the VEGF gradient to the retina without blocking the physiological level of VEGF in the retina, to maintain physiological vascularization and block the intravitreal vasoproliferation. John Penn (10) reported that VEGF levels were significantly increased in the vitreous at p18. For this reason, we planned our treatments at p18 when the vessels begin to migrate from the retinal surface to the vitreous due to the VEGF gradient to block the vasoproliferative process in the vitreous, while the physiological retinal vascularization continues.

There are no reports on the pharmacokinetics of rat anti-VE$\mathrm{GF}_{164} \mathrm{Ab}$ after SC injection, but a similar study on ranibizumab, a human anti-VEGF Ab, was published by Zhao., et al [16]. The authors reported that the amounts of drug in the vitreous were 389.67 $\mathrm{mg} / \mathrm{ml}$ and $53.67 \mathrm{mg} / \mathrm{ml}$, respectively, one week after the same doses of IVT and SC injections into rabbit eyes. After two weeks, the amounts of drugs from the IVT and SC injections were 319.33 and $12.67 \mathrm{mg} / \mathrm{ml}$, respectively. Within two weeks, Zhao., et al. observed that the range of the drug concentration in the vitreous after the SC and IVT injections varied between $1 / 7$ and $1 / 27$, respectively. Although this study was conducted in rabbits, the data in this study guided our SC treatment. In our study, since the follow-up period after injections was approximately two weeks, we determined that the SC dose should be 20 times higher than the IVT dose.

The MRSS changes in G2 showed that the severity of retinopathy slightly decreased in the p18-p19 interval and then increased slightly in the p19-p25 interval. Neither the p18-p19 nor the p19-p25 intervals were statistically significant. However, a significant decrease in the severity of retinopathy occurred in the p25-p30 interval. This result means that the resolution of OIR approximately begins in the p25 - p30 interval in the rat OIR model, and this result was similar to the results of many previous studies [11-14]. A significant decrease in the MRSS between the p18-p19 interval showed that therapeutic effects of the SC and IVT injections began to appear one day after injection. This effect continued and slightly increased between the p19-p25 interval and then became more powerful in the p25-p30 interval. Although the therapeutic effect of the IVT treatment was stronger than SC treatment from first day to the end of the study, this difference did not reach a statistically significant point at any time during our study. We think that this was one of the most striking findings of our study. Additionally, the therapeutic effect of both the SC and IVT treatments began with-in 24 hours of the injections. The most notable finding in this study was that there was no significant difference between G3 and G4 in any time interval. We think, that the similarities between the rapid effects of the SC and IVT injections were due to the rapid absorption of drug from the subconjunctival and episcleral spaces to the vitreous and retina.

We observed that the systemic VEGF levels in healthy rat pups were significantly lower than those in all the OIR groups, which we expected. In the OIR groups, the systemic VEGF level was approximately 1.5 - 2 times higher than in the healthy group. In addition to this finding, in all the OIR groups, there was a statistically significant decrease in the p19-p25 interval. We think that, this result was due to the natural decrease of the OIR-related high systemic VEGF level in G2. However, in G3 and G4, this decrease was associated with a systemic VEGF blockade effect induced by the rat anti-VEGF $\mathrm{Ab}$ in addition to the natural decrease in the OIR-related high systemic VEGF levels. In our study, we observed that this effect decreased systemic VEGF levels below the level of healthy rat pups at approximately before p25 until p30. We thought that systemic side effects of the SC and IVT anti-VEGF Ab injections would occur due to this extreme decrease in systemic VEGF levels at this critical time interval. We observed that, the systemic VEGF-blocking effects of the treatments started between p19 - p25, and these effects were correlated with the therapeutic effect on the vitreous and retina at the same time. It is crucial that the systemic VEGF levels were approximately at the same level at p30. Although the SC treatment is potentially disadvantageous in terms of dose and volume in our study, there was no significant difference in terms of the systemic VEGF blockade effect, which is an important factor for systemic side effects. In light of these data, we conclude that there is no difference between the two treatments in terms of the complications that may occur through systemic VEGF blockade after the SC and IVT treatments. 
Evaluation of the Efficacy of Subconjunctival Anti Vascular Endothelial Growth Factor İnjection in the Treatment of Retinopathy of Prematurity in an Experimental Rat Model

In the histopathological examination, we did not detect any neovascular progression on retinal surface and vitreous of healthy rat pups in any time interval. However, in all the OIR groups, there were NVs on the retinal surface in all time intervals. Additionally, in G2, we also detected vascular lumens in the vitreal space. In addition to this result, there were no vascular lumens in the vitreous in G3 and G4 in any time interval. We think that injections at p18 when the VEGF gradient had begun to increase in the vitreous prevented the neovascular progression in vitreous space in G3 and G4. A statistically insignificant reduction in the number of vascular lumens on the retinal surface in G2 suggests that the disease was minimal and mostly resolved by this time. The neovascularization blocking effect of the SC treatment reached a significant point at the second week in our study. The effectiveness of the IVT treatment on NV started between the p19 - p25 interval and continued until p30. When G3 and G4 were compared, the IVT treatment was superior to the SC treatment in terms of efficacy for reducing NV, but this difference was not statistically significant in our study.

Although the SC injection appears less effective than the IVT injection in terms of the histopathological results $\mathrm{NV}$ is only one of the characteristic features of ROP, and ROP also involves other pathologies in both the acute and chronic stages, which were used as criteria for the MRSS in our study. The MRSS results shows that the SC treatment is as effective as IVT treatment in OIR rat pups.

\section{Conclusion}

In conclusion, the SC injection of the rat anti-VEGF ${ }_{164} \mathrm{Ab}$ is effective for the treatment of the rat OIR model, and this effect is very close to the IVT treatment. The systemic VEGF blocking effects of SC and IVT injections are similar. This similarity suggests that there is no disadvantage for the SC treatment in terms of complications arising from systemic VEGF blockade compared to the IVT treatment. It is also clear that the likelihood of the SC injections leading to ocular complications is much less than that of the IVT treatments. We think that the likelihood of the SC injections leading to ocular complications is much less than that of the IVT treatments due to no penetration into the globe. In light of this information, if the results of our work are supported by further studies on a large number of animals and humans, we think that SC injection may be a safer and effective alternative to IVT injections for the treatment of ROP in the future.

\section{Funding}

Revolving Fund Board of Gülhane Military Medical Academy Haydarpaşa Training and Research Hospital.

\section{Conflict of Interest}

None.

\section{Bibliography}

1. Pozarowska D and Pozarowski P. "The era of anti-vascular endothelial growth factor (VEGF) drugs in ophthalmology, VEGF and anti-VEGF therapy". Central-European Journal of Immunology 41 (2016): 311-316.

2. Connolly BP MJ., et al. "A comparison of laser photocoagulation with trans-scleral cryotherapy in the treatment of threshold retinopathy of prematurity". Ophthalmology 105 (1998): 1628-1631.

3. Jandeck C. "[New therapeutic approaches in the treatment of retinopathy of prematurity]". Klinische Monatsblatter Fur Augenheilkunde 226 (2009): 914-920.

4. Karkhaneh R., et al. "Efficacy of intravitreal bevacizumab for zone-II retinopathy of prematurity". Acta Ophthalmology 94 (2016): 417-420.

5. Geloneck MM CA., et al. "Refractive outcomes following bevacizumab monotherapy compared with conventional laser treatment: a randomized clinical trial". JAMA Ophthalmology 132 (2014): 1327-1333.

6. O'Keeffe N., et al. "Bevacizumab compared with diode laser in stage 3 posterior retinopathy of prematurity: A 5 year follow up". Irish Medical Journal 109 (2016): 355.

7. Larranaga-Fragoso P., et al. "Intravitreal Bevacizumab for Zone II Retinopathy of Prematurity". Journal Of Pediatric Ophthalmology And Strabismus 53 (2016): 375-382.

8. Mintz-Hittner HA., et al. "Efficacy of intravitreal bevacizumab for stage 3+ retinopathy of prematurity". The New England Journal of Medicine 364 (2011): 603-615.

9. Xu Y and Tan CS. "Safety and complications of intravitreal injections performed in an Asian population in Singapore". International Ophthalmology (2016). 
10. Penn JS HM and Tolman BL. "Exposure to alternating hypoxia and hyperoxia causes severe proliferative retinopathy in the newborn rat". Pediatric Research 36 (1994): 724-731.

11. Liu K., et al. "The retinal vasculature and function of the neural retina in a rat model of retinopathy of prematurity". Investigative Ophthalmology and Visual Science 47 (2006): 2639-2647.

12. Hartnett ME., et al. "Neutralizing VEGF decreases tortuosity and alters endothelial cell division orientation in arterioles and veins in a rat model of ROP: relevance to plus disease". Investigative Ophthalmology and Visual Science 49 (2008): 31073114.

13. Werdich XQ and Penn JS. "Specific involvement of SRC family kinase activation in the pathogenesis of retinal neovascularization". Investigative Ophthalmology and Visual Science 47 (2006): 5047-5056.

14. Akula JD., et al. "The anatomy of the rat eye with oxygen-induced retinopathy". Documenta Ophthalmologica Advances In Ophthalmology 120 (2010): 41-50.

15. Geisen P PL., et al. "Neutralizing antibody to VEGF reduces intravitreous neovascularization and does not interfere with vascularization of avascular retina in an ROP model". Molecular Vision 14 (2008): 718-727.

16. Zhao $\mathrm{Y}$ and Lei W. "[Retina penetration of subconjunctival ranibizumab injection in the rabbit eye]". [Zhonghua Yan Ke Za Zhi] Chinese Journal Of Ophthalmology 51 (2015): 356-359.

\section{Assets from publication with us}

- Prompt Acknowledgement after receiving the article

- Thorough Double blinded peer review

- Rapid Publication

- Issue of Publication Certificate

- High visibility of your Published work

Website: $\underline{w w w}$.actascientific.com/

Submit Article: www.actascientific.com/submission.php

Email us: editor@actascientific.com

Contact us: +919182824667 